\title{
Broadband Waveguide QED System on a Chip
}

\section{Citation}

Quan, Qimin, Irfan Bulu, and Marko Loncar. 2009. Broadband waveguide QED system on a chip. Physical Review A 80(1): 1-4.

\section{Published Version}

http://dx.doi.org/10.1103/PhysRevA.80.011810

\section{Permanent link}

http://nrs.harvard.edu/urn-3:HUL.InstRepos:3409103

\section{Terms of Use}

This article was downloaded from Harvard University's DASH repository, and is made available under the terms and conditions applicable to Other Posted Material, as set forth at http:// nrs.harvard.edu/urn-3:HUL.InstRepos:dash.current.terms-of-use\#LAA

\section{Share Your Story}

The Harvard community has made this article openly available.

Please share how this access benefits you. Submit a story.

\section{Accessibility}




\title{
Broadband waveguide QED system on a chip
}

\author{
Qimin Quan, Irfan Bulu, and Marko Lončar \\ School of Engineering and Applied Science, Harvard University, Cambridge, Massachusetts 02138, USA
}

(Received 22 January 2009; published 28 July 2009)

\begin{abstract}
We demonstrate that a slot waveguide provides a broadband loss-free platform suitable for applications in quantum optics. We find that strong coupling between light quanta and a single quantum emitter placed in the waveguide slot can be achieved with efficiency higher than $96 \%$ and Purcell factor (spontaneous emission factor) larger than 200. The proposed system is a promising platform for quantum information processing and can be used to realize an efficient single photon source and optically addressable photon register.
\end{abstract}

DOI: $10.1103 /$ PhysRevA.80.011810

PACS number(s): 42.79.Gn, 42.50.Ct, 42.50.Ex, 78.67.Hc

Photons have vanishingly small cross sections for direct interaction and therefore they need to be mediated through interactions with matter [1]. Strong interactions can be achieved by placing the atom into structured photon "reservoir" such as optical cavities [2-9], photonic crystal waveguides [10-12], and metal nanowires $[13,14]$. This regime, so-called strong coupling regime, allows for the emission of photons into a desired optical channel and preserves the coherence between the photon and atom. Cavity based system for quantum electrodynamics (cQED) benefits from small photon loss rate due to the high quality factor $(\mathrm{Q})$ of the cavity. At the same time, such systems suffer from the small bandwidth of the resonance and require a tuning mechanism to match the cavity and emitter resonances [15]. Photonic crystal waveguides can provide wider bandwidths but an intrinsic tradeoff between the coupling strength and bandwidth exists, since the former depends on a large local density of states (LDOS) provided by guided modes with small group velocity ("flat band modes"). The metallic nanowire approach takes advantage of the subwavelength localization of surface plasmons and is broadband in nature, but it suffers from large propagation losses. Optical waveguides have also been studied in the context of broadband photon extraction from proximal quantum emitters [16,17], but with modest efficiency $\approx 30 \%$. Recently, Lecamp and co-workers [10] theoretically demonstrated that the extraction efficiency can be larger than $90 \%$ when the emitter is placed inside a cylindrical nanowire, while the spontaneous emission rate is similar to the emission rate in the bulk medium [18].

In this work, we propose a cavity-free, loss-free, and broadband slot waveguide-based QED (wQED) system on chip (Fig. 1), in which the single emitter is strongly coupled to the waveguide mode. We emphasize that a large bandwidth of strong coupling is beneficial for spectroscopy applications [19,20], multicolor information processing [21], etc. In our system all the information of the atomic states is coherently extracted into the waveguide mode with high fidelity. At the same time, coherent oscillations between photonic state and atomic state do not exist due to the lack of the optical resonance. The proposed wQED takes advantage of ultrasmall mode size provided by a slot waveguide. We demonstrate that this system is a promising platform for quantum information processing $[22,23]$ and can be used to realize an efficient single photon source and optically addressable single photon register.

The system that we are interested in (Fig. 1) is based on a slot-waveguide geometry [24-35]. In this work, we focus on two quantities to characterize the coupling between the waveguide and quantum emitters placed in the slot, namely, (i) the emission enhancement parameter $\alpha=\gamma_{\mathrm{wg}} / \gamma_{0}$ as the ratio between the emission rate into waveguide mode (both directions combined) and the intrinsic spontaneous emission rate in free space, and (ii) the waveguide coupling efficiency $\beta=\gamma_{\mathrm{wg}} / \gamma_{\text {total }}$, as the ratio between the emission rate into the waveguide mode (both directions combined) and total emission rate into all radiative channels. We note that figure of merit $\beta /(1-\beta)$ corresponds to cooperativity often used in cQED (that is, optical depth used in cold atoms) and it discriminates the strong coupling from weak coupling regime. In the former case, highly nonlinear optical effects are expected [36], which are important for devices such as a single atom quantum gate [37] and a single photon transistor [38]. The total enhancement of spontaneous emission, the Purcell factor [39], can be found as $\alpha / \beta$.

This emitter-waveguide system can be described in a way similar to Wigner-Weisskopf theory. The quantum emitter is coupled to both the continuum modes of free space and to the modes of the slot waveguide. The coupling strength to each waveguide mode is denoted by $g_{k}^{i}$, where $i$ denotes mode index and $k$ the effective wave vector. With $\vec{\phi}$ being the electric field, the transverse mode size can be defined as $S=\int \epsilon_{r}|\vec{\phi}|^{2} d \mathbf{r}_{T} /\left(\epsilon_{r}|\vec{\phi}|^{2}\right)_{\max }$ and plays the role analogous to the mode volume used in $\mathrm{CQED}$. The atom spontaneously radiates into a particular waveguide mode with a rate of $\gamma_{\mathrm{wg}}^{i}$ $=2\left|g_{k}^{i}\left(\omega_{k}^{i}=\omega\right)\right|^{2} L / v_{g}^{i}[40]$, where $L$ is the quantization length and $v_{g}^{i}=d \omega_{k}^{i} / d k$ is the group velocity of that mode. The spontaneous emission rate into free space is $\gamma_{0}=\frac{\mu^{2} \omega^{3}}{3 \pi \epsilon_{0} c^{3} \hbar}$ and the enhancement is

$$
\alpha=\frac{\gamma_{\mathrm{wg}}}{\gamma_{0}}=\sum_{i} \frac{3}{4 \pi} \frac{\lambda_{0}^{2}}{S^{i}} \frac{c}{v_{g}^{i}} \frac{\left|\vec{\phi}^{i}\left(\mathbf{r}_{0}\right) \cdot \hat{\mu}\right|^{2}}{\left|\left(n \vec{\phi}^{i}\right)_{\max }\right|^{2}} .
$$

The sum is over all the modes that the slot waveguide supports; $\mathbf{r}_{0}$ represents the position of the emitter. Clearly, if the mode size $S$ is squeezed significantly below $\lambda_{0}^{2}$, then the light-matter interaction could be dramatically enhanced. In addition, a reduction in the group velocity in a slot waveguide (group index $n_{v g} \sim 3.5-4$ ) increases the LDOS and also contributes to the increase in $\alpha$. However, this is a minor 


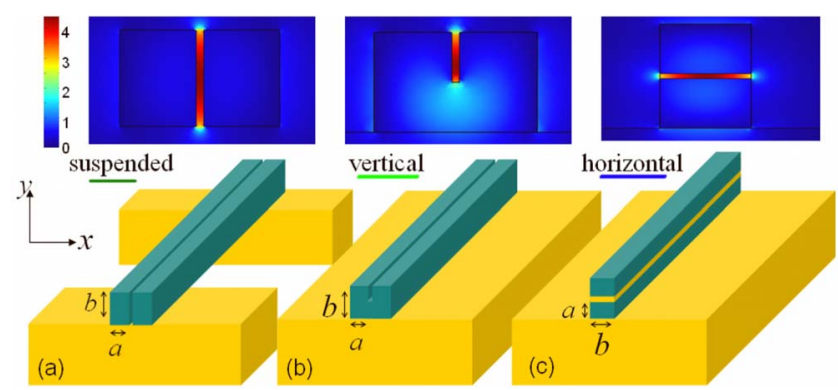

FIG. 1. (Color online) Configurations of proposed waveguide QED device. In all cases $\lambda=1.5 \mu \mathrm{m}$, and $a=0.2 \mu \mathrm{m}, b=0.25$ $\mu \mathrm{m}$. (a) A slot waveguide with material index 3.5 suspended in air. (b) Vertical and (c) horizontal on-substrate configurations. Insets show major field components $\left|E_{x}\right|$ in (a) and (b) and $\left|E_{y}\right|$ in (c).

effect compared to the enhancement due to light localization in the waveguide slot.

In order to capture the physical mechanism of the enhancement $\alpha$, we analyze a simple toy model that consists of a solid-core (slot width is zero) dielectric waveguide (index $n$ ), with cross section $a \times b$, surrounded by air. Neglecting the field outside the dielectric and applying a perfect electric conductor boundary condition, the mode inside the dielectric can be approximated by $\phi_{0}=\cos (\pi x / a) \cos (\pi y / b)$ resulting in the mode size $S=a b / 4$. We assume that the introduction of an air slot (width $\delta$ ) results only in the change in the field magnitude outside the slot, while overall mode shape is maintained, i.e., $\phi=t \phi_{0}(t$ is a coefficient which drops off in the final expression). This assumption is later validated by finite element calculations (Fig. 2). Within the slot, however, the electric field (TE-like polarized) can be written as $\phi$ $=t \epsilon \phi_{0}\left(\epsilon=n^{2}\right)$ due to boundary conditions. The mode size of the slot waveguide is then $S=a b /(4 \epsilon)+\delta b$, where $\delta$ is the width of the slot. Hence the ratio between the slot and the solid-core waveguide mode size is $\eta(\delta)=\frac{a / 4+\epsilon \delta}{\epsilon a / 4} \rightarrow \frac{1}{\epsilon}$, as $\delta$ $\rightarrow 0$. The results from the toy model, presented in the inset table in Fig. 2, are in good agreement with the numerical

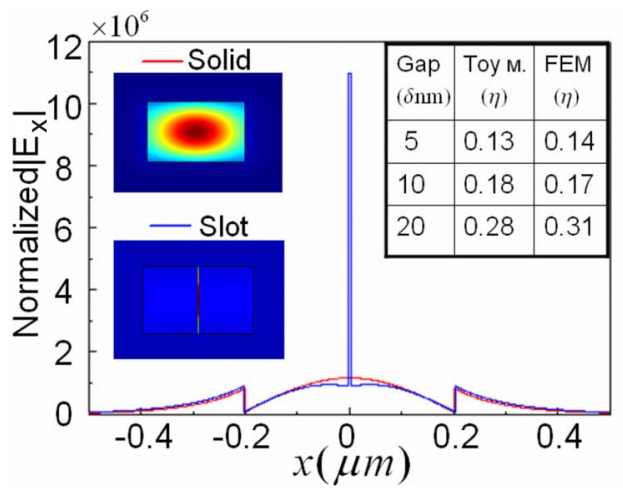

FIG. 2. (Color online) Comparison between $E_{x}$ for solid-core waveguide and slot waveguide with gap width $5 \mathrm{~nm}$, normalized to a single photon, i.e., $\left|E_{x}\right| / \sqrt{\int \epsilon_{r}\left|\mathbf{E}\left(\mathbf{r}_{T}\right)\right|^{2} d \mathbf{r}_{T}}$. For slot waveguide, $\left.E_{x}\right|_{x=0}$ directly reflects the inverse of mode size. Inset plots are the electric energy density of each mode. Inset table shows the comparison between $\eta$ obtained using a toy model and full-vectorial FEM calculation.
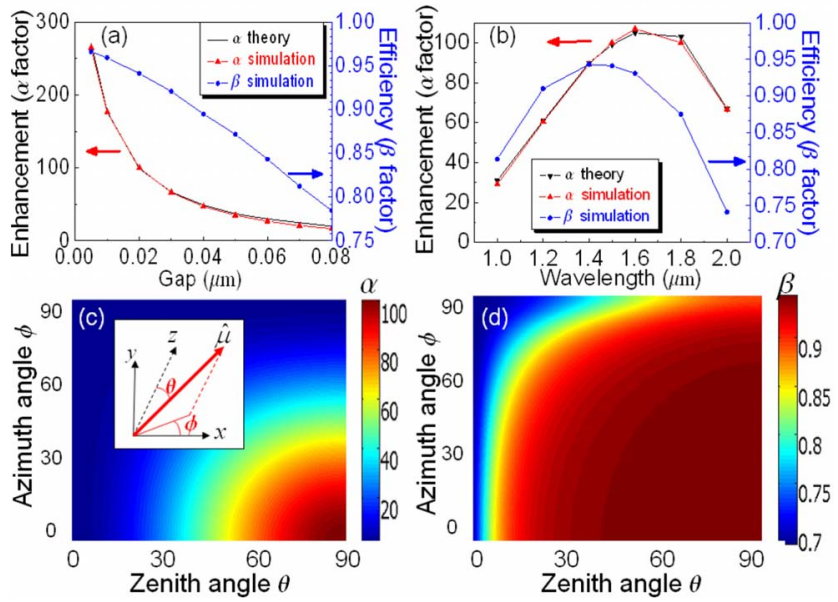

FIG. 3. (Color online) (a) Enhancement ( $\alpha$ factor) and efficiency ( $\beta$ factor) for slot waveguide with $a=0.2 \mu \mathrm{m}$ and $b=0.25 \mu \mathrm{m}$ at different gap sizes. The dipole emits at $1.5 \mu \mathrm{m}$. (b) $\alpha$ and $\beta$ factors vs wavelength. (c) and (d) show the polarization dependence of $\alpha$ and $\beta$ factors, respectively. Inset (c): the zenith angle is between the dipole orientation and the $z$ axis, and azimuthal angle is between the $x$-axis and projection of the dipole orientation onto the $x-y$ plane.

calculations using finite element method (FEM). Since $n=1$ at $(n \vec{\phi})_{\max }$, the enhancement emission rate provided by the slot waveguide, compared to the free space, scales as $\alpha$ $\propto \epsilon^{2} \cdot n_{v g}$, and this scaling law is strictly valid only at the zero slot limit. A similar scaling was obtained by Ho et al. [27] who studied the inhibition of spontaneous emission. In comparison, solid-core waveguide provides an enhancement of $n_{v g}$ and homogenous bulk medium enhances by $n_{\text {bulk }}$ over the free space emission rate.

The exact quantitative value of the $\alpha$ factor can be calculated in two ways: (i) from expression (1) using the field profile from finite element method and (ii) directly from 3D finite-difference time-domain (FDTD) simulation by placing a dipole source at the center of the gap and monitoring the power emitted into the waveguide. The $\beta$ factor can be obtained using this latter approach as well. Figure 3 shows the $\alpha$ and $\beta$ factors of the slot waveguide with $a=0.2 \mu \mathrm{m}$ and $b=0.25 \mu \mathrm{m}$ for different gap widths, different wavelengths, and different polarizations. In Fig. 3(a), we assumed an $\hat{x}$-polarized dipole source that emits at $1.5 \mu \mathrm{m}$, placed at the center of the slot and varied the gap from 5 to $80 \mathrm{~nm}$. An excellent agreement between $\alpha$ 's obtained using expression (1) and FDTD simulation is obtained. An enhancement of up to 270 is obtained for a gap size of $5 \mathrm{~nm}$, and 100 is obtained with a moderate gap size of $20 \mathrm{~nm}$. An efficiency better than $90 \%$ is maintained for a gap width $\leq 30 \mathrm{~nm}$. As the slot width is further reduced toward zero width, $\alpha$ factor converges to $3 \epsilon^{2} n_{v g} / \pi$ assuming that corresponding solid-core waveguide has a minimum mode size of $\left(\lambda_{0} / 2 n\right)^{2}$. This upper limit, however, is valid within mesoscopic scale when materials can be described by different refractive indices and the Maxwell boundary conditions are valid. For vanishingly small slots (down to the atomic scale), the discontinuity of boundary conditions for $E$ field breaks down and one recovers the solid-core waveguide regime. Figure 3(b) shows the enhancement factor at different wavelengths for a slot wave- 
guide with $a=0.2 \mu \mathrm{m}, b=0.25 \mu \mathrm{m}$, and a moderate gap width $0.02 \mu \mathrm{m} . \alpha>50$ (half of its maximum value) is achieved in the wavelength range from 1.2 to $2 \mu \mathrm{m}$. The optimal value of $\alpha$ at $1.6 \mu \mathrm{m}$ is due to the tradeoff between the increase in wavelength and reduction in the effective mode index for longer wavelengths. The enhancement bandwidth for the current wQED system is over $800 \mathrm{~nm}$ and strong coupling is maintained across the wavelength range 1-2 $\mu \mathrm{m}$. As a comparison, a cQED system with a $Q$ $\sim 10000$ operating at $1.5 \mu \mathrm{m}$ has a bandwidth $\sim 0.15 \mathrm{~nm}$. However, a cQED system provides much larger values of $\alpha$ (larger than 1000 has been predicted). At the same time, the photonic crystal waveguide approach provides $\alpha \sim 30$ and $\beta \sim 0.9$ over a bandwidth of $20 \mathrm{~nm}$ [10-12]. Finally, solidcore waveguide, with core index $n$ and air cladding, provides $\alpha_{\text {solid }} \sim n$, which is similar to the enhancement in bulk material with the same index [18]. Therefore, the emission enhancement provided by the slot waveguide, when compared to both bulk and solid-core geometry, is approximately $\alpha_{\text {slot }} / n$ and is in the range of 10-100 for slot widths explored in Figs. 3(a) and 3(b). It is important to mention that slot geometry does not provide better waveguide coupling efficiencies, $\beta$, than solid-core geometry [18]. This can be attributed to the enhanced emission into the leaky modes of the slot waveguide, in addition to the guided ones. However, an advantage of the slot waveguide is its large Purcell factor $(\alpha / \beta)$ which enhances radiative processes over the nonradiative ones (common to solid-state emitters). This is highly desirable in order to coherently extract photons into the waveguide mode.

Figures 3(c) and 3(d) show the polarization dependence of the $\alpha$ and $\beta$ factors for a slot waveguide with a $20 \mathrm{~nm}$ gap. This waveguide geometry supports one TE-like mode with phase index $1.68\left[E_{x}\right.$ profile shown in Fig. 1(a)] along with two other modes (effective indices of 1.49 and 1.03) with different symmetries (not shown). Differently polarized dipoles will preferentially couple to one of these three modes. For the $\hat{y}$ or $\hat{z}$ dipole, there is virtually no emission enhancement $(\alpha \sim 1)$. Therefore, the amount of spontaneous emission rate enhancement can be viewed as a contribution from the $\hat{x}$ component of the dipole. The efficiencies of $\hat{y}$ and $\hat{z}$ dipoles are $89 \%$ and $69 \%$, respectively. Therefore, high extraction efficiency as well as strong coupling between an emitter and a slot waveguide is possible for range of different polarizations.

The slot waveguide is a convenient platform from the experimental point of view, since the emitter can be placed in the gap region where optical field is the strongest. This geometry can be used in QED experiments with cold atoms trapped directly by the waveguide field inside the slot [41] or in a solid-state QED system with quantum emitters (e.g., nanocrystals and molecules) placed in the slot. We show two experimental schemes for a solid-state QED application, namely, the vertical half-slot and horizontal slot in Figs. 1(b) and $1(\mathrm{c})$. In the vertical configuration, the slot is etched only halfway and the quantum emitter is placed at the bottom (center of the waveguide). In the horizontal configuration, the quantum emitters are embedded within the low index material that forms the slot $(n=1.46)$ [29]. For these two realistic cases, we estimate the degradation of the $\alpha$ and $\beta$

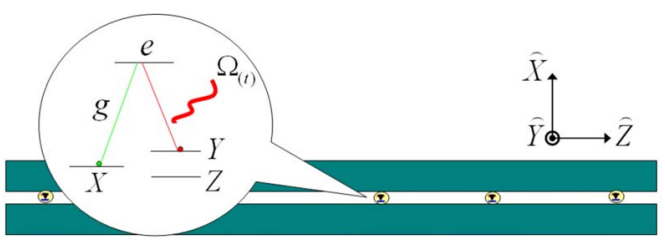

FIG. 4. (Color online) A schematic of optically addressable photon register that allows storage (retrieval) of a photon into (out of) a specific quantum emitter. Before and after the storage (retrieval), the emitter is decoupled from the waveguide.

factors due to reduced index contrast by simulating the nanocrystal quantum dot as a sphere with diameter of $10 \mathrm{~nm}$ with refractive index $n=2$ (average of core and shell in $\mathrm{PbS}$ quantum dots). We do not take into account the details of the inner structure of solid-state emitters or local field correction due to various field-screening effects [42]. We find that $\beta$ $\sim 0.91$ for the horizontal configuration and $\beta \sim 0.72$ for the vertical configuration, for geometries in the range $a$ $\in[0.2,0.3] \mu \mathrm{m}, b \in[0.2,0.3] \mu \mathrm{m}$, and gap width $=20 \mathrm{~nm}$. We also find $\alpha_{\text {hoz }}=98$ and $\alpha_{\text {ver }}=28$, when compared to a dipole embedded in a $10 \mathrm{~nm}$ diameter nanocrystal with index of $2\left(\alpha_{\mathrm{hoz}}=14\right.$ and $\alpha_{\mathrm{ver}}=4$, when compared to a dipole embedded in a uniform medium with index of 2). Reduction in the enhancement is due to smaller index contrast between waveguide and emitter as well as nonideal overlap between the field and emitter in the vertical case. However, the efficiency is robust and does not depend strongly on the effective index of the emitter but instead on the waveguide index.

The impressive ability to enhance the emission and extraction of photons with high fidelity makes the slot waveguide an appealing platform for applications in quantum information. Here we propose an implementation of an optically addressable photon register shown in Fig. 4. Single emitters, used to store photons, are placed along the waveguide so that each can be addressed individually using external control pulses (can be integrated on the same chip). We assume that each emitter has a triplet ground state, denoted according to polarization as $X, Y$, and $Z$ states, and $e$ is the excited state. For the TE-like mode, the $\hat{X}$ component is the major component and is the only nonvanishing component at the center of the slot. As a result, emitters polarized in $\hat{Y}$ or $\hat{Z}$ direction will not couple to the TE-like slot waveguide mode. Our register is first initialized by setting all emitters into the $Z$ state, so that they are decoupled from the waveguide and do not interact with light propagating down the waveguide. Next, one of the emitters is prepared by external control into the $X$ state without changing the states of the other units. Now, a single photon propagating in the waveguide can be absorbed by the emitter, thus setting the emitter into the state $Y$. This can be achieved by applying a time-dependent classical control pulse on transition between $e$ and $Y$ state, using techniques similar to the ones originally used by Fleischhauer et al. $[43,44]$ to map single photon field to collective atomic excitations. Once the photon is stored in $Y$ state the unit is again decoupled from the waveguide. Photon retrieval can be accomplished using the same control pulse, time reversed. In this case, the population starts from $Y$ state and 
evolves as $\quad c_{Y}(t)=e^{-\gamma(t, 0)}$, with $\quad \gamma(t, 0) \triangleq \int_{0}^{t} d t 2|\Omega(t)|^{2}$ $/\left(\gamma_{\mathrm{wg}}+\gamma^{\prime}\right)$. Retrieval efficiency is $\beta\left(1-e^{-\gamma(\infty, 0)}\right) \rightarrow \beta$. Here, $\Omega(t)$ is the Rabi frequency of the classical control, $\gamma_{\mathrm{wg}}$ is the emission rate into waveguide (both directions), and $\gamma^{\prime}$ is the decay rate into other channels. The outgoing photon splits equally into a left-propagating and a right-propagating pulse, and its shape is determined by the control pulse $c_{ \pm}^{\text {out }}$ $\propto \theta( \pm z) \theta\left(t \mp z / v_{g}\right) \Omega\left(t \mp z / v_{g}\right) \exp \left[-\gamma\left(t \mp z / v_{g}, 0\right)\right] \exp [ \pm i \omega z$ $\left./ v_{g}\right], \theta$ is the Heaviside function, $v_{g}=d \omega_{k} / d k$ is the group velocity, and $\omega$ is the energy difference between $e$ and $X$ state. The storage process can be understood as a time reversal of the retrieval process. Let the incoming photon shape at $z=0$ (position of the emitter) be $c^{\text {in }}(z=0, t)$. In order to maximize $c_{Y}(t=\infty)$, the control pulse should then take the form $\Omega(t)=\frac{\sqrt{\gamma_{\mathrm{wg}}+\gamma^{\prime}}}{2} \frac{\left|c^{i n}\left(t^{\prime}\right)\right|}{\left[\int_{0}^{t} d t^{\prime}\left|c^{i n}\left(t^{\prime}\right)\right|^{2}\right]^{1 / 2}}$. The storage efficiency $\rightarrow \beta$ when the incoming photon is split into two symmetric pulses arriving from both sides. Otherwise, if the incoming photon arrives from one side only, the efficiency is limited to $50 \%$. This is a consequence of the waveguide symmetry which results in emitter coupling into the symmetric $a_{R k}^{\dagger}+a_{L-k}^{\dagger}$ state only, while the antisymmetric $a_{R k}^{\dagger}-a_{L-k}^{\dagger}$ state is a dark state.

The proposed scheme can also be used to implement a quantum memory: due to the strong coupling between a waveguide mode and an atom placed in the slot, the atomic state $\cos \theta|Y\rangle+e^{i \phi} \sin \theta|X\rangle$ can be efficiently mapped onto the photon state (using classical control pulse) $\cos \theta|1\rangle$ $+e^{i \phi} \sin \theta|\mathrm{vac}\rangle$, with high fidelity. Our platform can also be used to realize single-photon transistors similar to the one based on metallic nanowires recently proposed by Chang et al. [38]. The advantage of the slot waveguide, however, is that the material losses are negligible.

In conclusion, we have proposed a broadband, loss-free, on chip slot-waveguide QED system that can operate at high photon collection efficiency $\beta \sim 94 \%$ (corresponding to cooperativity of 16) and a spontaneous emission enhancement of $\alpha \sim 100$, at a moderate slot width of $20 \mathrm{~nm}$. We have also shown that, in experimentally more accessible configurations, high coupling efficiencies can be achieved. The broadband nature of wQED system makes it an attractive alternative to CQED since it does not require any tuning mechanism and it could be beneficial for applications in spectroscopy and multicolor information processing.

The authors acknowledge helpful discussions with L. Jiang, J. T. Robinson, J. Choy, and A. S. Zibrov. This work was supported by NSF NIRT Grant No. ECCS-0708905.
[1] B. Dayan et al., Science 319, 1062 (2008).

[2] T. Aoki et al., Nature (London) 443, 671 (2006).

[3] C. Santori et al., Nature (London) 419, 594 (2002).

[4] J. P. Reithmaier et al., Nature (London) 432, 197 (2004).

[5] T. Yoshie et al., Nature (London) 432, 200 (2004).

[6] E. Peter et al., Phys. Rev. Lett. 95, 067401 (2005).

[7] K. Hennessy et al., Nature (London) 445, 896 (2007).

[8] D. Englund et al., Nature (London) 450, 857 (2007).

[9] K. Srinivasan and O. Painter, Nature (London) 450, 862 (2007).

[10] G. Lecamp, P. Lalanne, and J. P. Hugonin, Phys. Rev. Lett. 99, 023902 (2007).

[11] V. S. C. Manga Rao and S. Hughes, Phys. Rev. Lett. 99, 193901 (2007).

[12] T. Lund-Hansen et al., Phys. Rev. Lett. 101, 113903 (2008).

[13] D. E. Chang, A. S. Sorensen, P. R. Hemmer, and M. D. Lukin, Phys. Rev. Lett. 97, 053002 (2006).

[14] D. E. Chang, A. S. Sorensen, P. R. Hemmer, and M. D. Lukin, Phys. Rev. B 76, 035420 (2007).

[15] A. Faraon et al., Appl. Phys. Lett. 92, 043123 (2008).

[16] V. V. Klimov and M. Ducloy, Phys. Rev. A 69, 013812 (2004).

[17] F. Le Kien, S. Dutta Gupta, V. I. Balykin, and K. Hakuta, Phys. Rev. A 72, 032509 (2005).

[18] I. Friedler et al., Opt. Express 17, 2095 (2009).

[19] G. Wrigge et al., Nat. Phys. 4, 60 (2008).

[20] M. Winger, A. Badolato, K. J. Hennessy, E. L. Hu, and A. Imamoglu, Phys. Rev. Lett. 101, 226808 (2008).

[21] H. Kamada, NTT Tech. Rev. 1, 31 (2003).

[22] L. M. Duan et al., Phys. Rev. A 73, 062324 (2006).

[23] L. Jiang, J. M. Taylor, A. S. Sorensen, and M. D. Lukin, Phys. Rev. A 76, 062323 (2007).
[24] V. R. Almeida et al., Opt. Lett. 29, 1209 (2004).

[25] Q. Xu et al., Opt. Lett. 29, 1626 (2004).

[26] J. T. Robinson, C. Manolatou, L. Chen, and M. Lipson, Phys. Rev. Lett. 95, 143901 (2005).

[27] S. T. Ho, S. L. McCall, and R. E. Slusher, Opt. Lett. 18, 909 (1993).

[28] T. Baehr-Jones et al., Appl. Phys. Lett. 86, 081101 (2005).

[29] R. Sun et al., Opt. Express 15, 17967 (2007).

[30] M. Hochberg et al., Opt. Express 15, 8401 (2007).

[31] J. T. Robinson et al., Opt. Express 16, 16659 (2008).

[32] C. C. Lin, D. G. Deppe, and C. Lei, IEEE J. Quantum Electron. 30, 2304 (1994).

[33] C. F. Janz and J. N. McMullin, IEEE J. Quantum Electron. 31, 1344 (1995).

[34] M. Galli et al., Appl. Phys. Lett. 89, 241114 (2006).

[35] C. Creatore et al., Appl. Phys. Lett. 94, 103112 (2009).

[36] See H. Mabuchi and A. C. Doherty, Science 298, 1372 (2002) and references therein.

[37] K. M. Gheri and H. Ritsch, Phys. Rev. A 56, 3187 (1997).

[38] D. E. Chang et al., Nat. Phys. 3, 807 (2007).

[39] E. M. Purcell, Phys. Rev. 69, 681 (1946).

[40] P. Domokos, P. Horak, and H. Ritsch, Phys. Rev. A 65, 033832 (2002).

[41] J. P. Burke, S. T. Chu, G. W. Bryant, C. J. Williams, and P. S. Julienne, Phys. Rev. A 65, 043411 (2002).

[42] G. L. J. A. Rikken and Y. A. R. R. Kessener, Phys. Rev. Lett. 74, 880 (1995).

[43] M. Fleischhauer, S. F. Yelin, and M. D. Lukin, Opt. Commun. 179, 395 (2000).

[44] A. V. Gorshkov, A. Andre, M. Fleischhauer, A. S. Sorensen, and M. D. Lukin, Phys. Rev. Lett. 98, 123601 (2007). 